\title{
Fix Smile for a While: An Esthetic Rehabilitation with Groper's Appliance - Two Case Reports
}

\author{
Manitha Pershad Seth, S. Thabitha Rani, Kiranmayi Merum, Srikanth Raju \\ Department of Paediatric and Preventive Dentistry, Kamineni Institute of Dental Sciences, Narketpally, Telangana, India
}

Email for correspondences: mani.manitha@gmail.com

\begin{abstract}
Esthetic rehabilitation of a preschooler with advanced carious lesions or a trauma is the mainstay of pediatric dental practice. Parental desire is the most decisive factor for the placement of an anterior esthetic appliance. The present article discusses about unique case reports highlighting the fabrication of Groper's appliance in a young child with early childhood caries and with trauma which is a simple technique for the placement of a fixed type of an anterior esthetic appliance. It constituted a design, whereby the maxillary primary second molars were used to support the appliance through bands and a wire that contained an acrylic flange bearing trimmed acrylic teeth, anteriorly. The appliance was functionally and esthetically compliant.
\end{abstract}

Key words: Esthetics, early childhood caries, fixed functional appliance, groper's appliance, trauma.

\section{INTRODUCTION}

Deciduous teeth are very inappropriately referred to as "temporary teeth" when in reality, they are responsible for general health, mastication, phonetics, esthetics and self-esteem, psychological comfort, etc. Deciduous teeth have an integral effect on the quality of life. Severe early childhood caries (S-ECC), if left untreated, progresses to severe destruction of teeth. Parents of these young children at this point of time overlook the clinical condition of the child relating to the shedding of primary teeth. ${ }^{[1]}$

Esthetic rehabilitation is one of the greatest challenges for a pediatric dentist's for treating a young toddler who has suffered multiple tooth loss subsequent to ECC or extensive dental trauma. ${ }^{[1]}$ When extraction of primary incisors is necessary, many parents will seek an esthetic solution to replace the lost teeth. Premature loss of anterior teeth in children has a far-reaching impact on the

\section{Quick Response Code Article Info:}

\begin{tabular}{|l|l|}
\hline & $\begin{array}{l}\text { doi: 10.5866/2018.10.10119 } \\
\text { Received: } 21-06-2018 \\
\text { Revised: } 25-07-2018 \\
\text { Accepted: } 21-08-2018 \\
\text { Available Online: } 25-09-2018,(w w w . ~ \\
\text { nacd.in)@ NAD, 2018 - All rights } \\
\text { reserved }\end{array}$ \\
\hline
\end{tabular}

psyche of the children. When these teeth are lost, replacement and prosthetic management is very important to restore all functions including esthetics of the child. The replacement should be such that it should not interfere with the eruption process of the underlying successor. Few esthetic options are available in removable or fixed partial dentures.

For the clinician seeking to construct and place an esthetic appliance in a preschooler, there is very little information in the dental literature which addresses the need or indications for these appliances. The present case reports discuss about unique case reports highlighting the fabrication of Groper's appliance in a young child with ECC and with trauma which is a simple technique for the placement of a fixed type of an anterior esthetic appliance.

\section{CASE REPORTS}

\section{Case 1}

A 6-year-old boy reported to the Department of Pedodontics, Kamineni Institute of Dental Sciences, with a chief complaint of pain in upper left and right back teeth region since a week. The parents were concerned about the esthetics of the child and wanted an esthetic replacement of the upper front teeth. On examination, no abnormality was detected extraorally and intraoral examination revealed caries irt. 51, 52, 54, 61, 62, 64, 74, 84, 

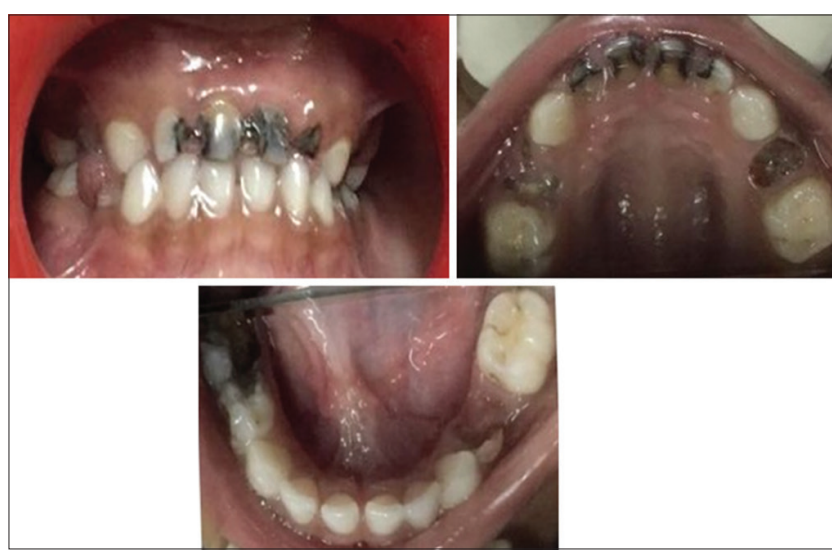

Figure 1: Clinical picture showing caries irt 51, 52, 54, 61, 62, $64,74,84$, and 85

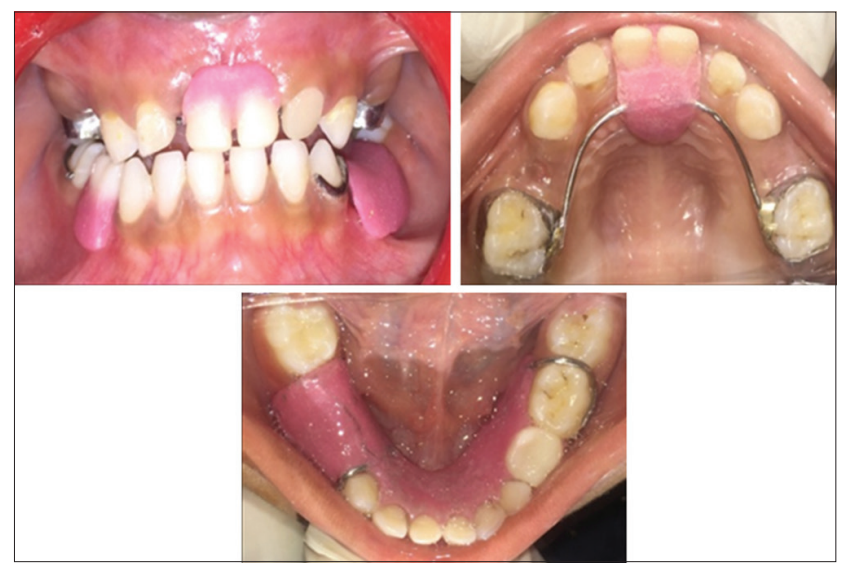

Figure 2: Post-operative picture showing Groper's appliance given in the maxillary arch irt 51,61 and removable space maintainer in the lower arch, functional irt 74 and nonfunctional irt 84, 85

and 85 [Figure 1]. The primary maxillary anteriors and maxillary and mandibular posteriors were grossly decayed. To evaluate the extent of pulpal involvement and status of root resorption, full mouth radiographs were taken. Radiographs revealed that $51,54,61,64,74,84$, and 85 teeth were grossly decayed, with severe root resorption. The grossly decayed teeth were planned for extraction and the extraction was carried out under LA, followed by fixed space maintainer irt 51and 61, and removable space maintainer being functional irt 74 and nonfunctional irt 84 and 85 . Composite buildup was done irt 52 and 62 following pulp therapy.

\section{Fabrication of Groper's appliance}

Considering the age and cooperation of the child, management approaches to esthetically

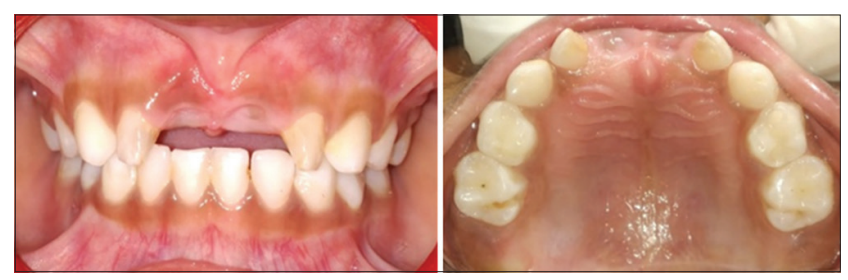

Figure 3: Clinical picture showing missing 51 and 61
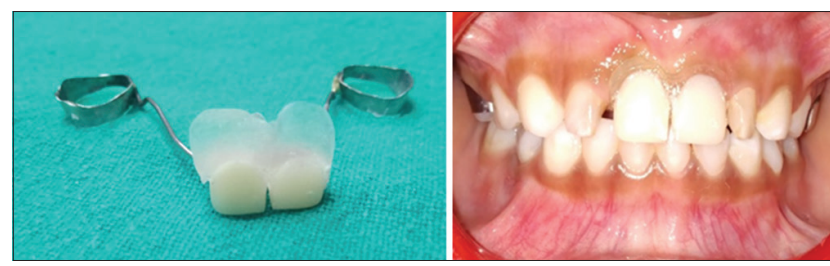

Figure 4: Post-operative picture showing Groper's appliance given in maxillary arch irt 51, 61

rehabilitate in premature loss of multiple primary anterior teeth are limited. Groper's appliance was chosen as it not only maintains the space but also esthetics. In the present case, 55 and 65 were chosen as abutments for the anterior space maintainer on which band adaptation was done. The primary impression was then made with irreversible hydrocolloid material - alginate. Dental casts were poured with Type III gypsum product - dental stone. On the upper cast, stainless steel framework was made from one side to the other (55 to 65), with an acrylic button similar to a Nance palatal arch with an extension of acrylic material being flowed from the palatal to the labial vestibule over the archwire. The wire was then soldered to the bands. Then, the acrylic teeth were taken and trimmed to the size of primary teeth; the teeth were placed directly on the alveolar crest with the acrylic material. Occlusion was then checked on the cast and then intraorally. After the necessary trimming and polishing, the appliance was cemented with glass ionomer cement Type I on 55 and 65 [Figure 2].

\section{Case 2}

A 5-year-old boy reported to the Department of Pedodontics, Kamineni Institute of Dental Sciences, with his parents with a chief complaint of missing upper front teeth. On examination, there was missing 51 and 61 [Figure 3]. The past dental visit revealed that the patient had a traumatic fall 6 months back due to which his teeth got mobile and were extracted in a clinic. The patient and the parent were very much concerned about the esthetics. Intraoral periapical radiograph was 
taken to find if any remnants are present followed by planning to replace the anterior esthetics with a fixed appliance. Similar Groper's appliance with clear acrylic was given [Figure 4].

\section{DISCUSSION}

The strongest factor for the placement of an anterior esthetic appliance is a parental desire. There is no strong evidence, suggesting that the early loss of the maxillary incisors will cause undesirable effects on the growth and development of the child..$^{[2]}$ However, considerations have to be given regarding the speech problems, masticatory inefficiency, abnormal oral habits, and a unesthetic appearance, which follow the loss of anterior teeth at an early age.

Loss of primary incisors after the eruption of primary canines is not an important consideration for space loss though occasionally in a crowded dentition, there may be a rearrangement of some anterior teeth. ${ }^{[3,4]}$ Another consideration is the child's speech development following extraction of primary incisors. The sounds most frequently in error are the labiolingual sounds. This is because many sounds are made with the tongue touching the lingual side of the maxillary incisors, and inappropriate speech compensations can develop if the teeth are missing. ${ }^{[5]}$

A study by Riekman and Badrawy reported that the loss of primary anterior teeth before the age of 3 years resulted in speech problems. ${ }^{[6]}$ One of the most considerable and valid reasons for replacing missing anteriors is to restore an esthetic appearance and thus promotes a normal psychological development in the child. The lingual sides of maxillary anterior teeth which are needed by the tongue for certain phonations and absence these teeth may result in improper speech. ${ }^{[3,6]}$ It usually affects sounds such as "s," "z," and "th." "[7] Gable et al. found that early loss of incisors had no long-term effects on speech. ${ }^{[8]}$

The factors associated with anterior tooth loss include tipping of adjacent teeth, overeruption of antagonist teeth, midline deviation, masticatory impairment, speech problems, and lingual dysfunction. ${ }^{[9]}$ When considering the need for an anterior appliance to replace missing primary incisors, a few points should be discussed with the parents. First, the strongest factor for placing this appliance is parental desire ${ }^{[2]}$ Here, in the present case, the patient had no complaints with mastication or speech, but they had complains with esthetics. This was probably because the patients had peer pressure in preschool.

This space maintainer offers several advantages in terms of esthetics, restoration of masticatory and speech efficiency, and prevention of abnormal oral habit development. The main disadvantage is the accumulation of food debris and plaque. Hence, parents have to be instructed to supervise the maintenance of proper oral hygiene in their child.

\section{CONCLUSION}

A simple technique for appliance placement was discussed. These appliances are almost always considered as an elective appliance and their placement is usually dictated by the wishes of the parent. The restoration of anterior esthetics and function with this appliance gave a huge psychological boost for the child. Oral hygiene instructions were given to the child and her parents.

\section{REFERENCES}

1. Ripa LW. Nursing caries: A comprehensive review. J Pediatr Dent 1988;10:268-82.

2. Waggoner WF, Kupietzky A. Anterior esthetic fixed appliances for the preschooler: Considerations and a technique for placement. Pediatr Dent 2001;23:147-50.

3. Christensen JR, Fields HW. Space maintenance in the primary dentition. In: Pinkham JR, editor. Pediatric Dentistry: Infancy through Adolescence. $2^{\text {nd }}$ ed. Philadelphia, PA: W.B. Saunders Company; 1994. p. 358-63.

4. Ngan P, Wei SH. Management of space in the primary and mixed dentitions. In: Itor Pediatric Dentistry: Total Patient Care. Philadelphia, PA: Lea and Febiger; 1988. p. 462-70.

5. Dyson JE. Prosthodontics for children. In: Wei SH, editor. Pediatric Dentistry: Total Patient Care. Philadelphia, PA Lea and Febiger; 1988. p. 259-74.

6. Riekman GA, el Badrawy HE. Effect of premature loss of primary maxillary incisors on speech. Pediatr Dent 1985;7:119-22.

7. Fymbo L. The relation of malocclusion of the teethto defects of speech. Arch Speech 1936;1:204-16.

8. Gable TO, Kummer AW, Lee L, Creaghead NA, Moore LJ. Premature loss of the maxillary primary incisors: Effect on speech production. ASDC J Dent Child 1995;62:173-9.

9. Liegeois F, Limme M. Modified bonded bridge space maintainer. J Clin Pediatr Dent 1999;23:281-4. 